\title{
Non-asymptotic information theoretic bound for some multi-party scenarios
}

\author{
Naresh Sharma and Naqueeb Ahmad Warsi \\ Tata Institute of Fundamental Research \\ Homi Bhabha Road, Mumbai 400005 \\ Email: \{nsharma, naqueeb\}@tifr.res.in
}

\begin{abstract}
In the last few years, there has been a great interest in extending the information-theoretic scenario for the non-asymptotic or one-shot case, i.e., where the channel is used only once. We provide the one-shot rate region for the distributed source-coding (Slepian-Wolf) and the multiple-access channel. Our results are based on defining a novel one-shot typical set based on smooth entropies that yields the one-shot achievable rate regions while leveraging the results from the asymptotic analysis. Our results are asymptotically optimal, i.e., for the distributed source coding they yield the same rate region as the Slepian-Wolf in the limit of unlimited independent and identically distributed (i.i.d.) copies. Similarly for the multiple-access channel the asymptotic analysis of our approach yields the rate region which is equal to the rate region of the memoryless multipleaccess channel in the limit of large number of channel uses.
\end{abstract}

\section{INTRODUCTION}

Over the last several decades, information theory has been used to analyze the performance of various information processing tasks such as data compression, information transmission across a noisy communication channel etc. The analysis has traditionally been asymptotic wherein a certain task is repeated unlimited number of times. Such an approach has yielded rich dividends providing fundamental limits to the performance and gave operational meaning to quantities such as entropy and mutual information [1], [2].

But the assumption of repeating an information processing task is hardly realistic. There has been a great interest over the last few years to analyze a task done only once. Typically, the analysis involves finding lower and upper bounds on the resources needed to perform such a task. This has been referred to as "one-shot" or "single-shot" in the literature.

The lower and upper bounds are expected to be asymptotically tight, i.e., they both yield the same average number of resources needed per task when the number of times a task is performed is unbounded and this quantity is also equal to the asymptotic analysis that has been done traditionally. It is interesting to note that while the asymptotic analysis would start with the asymptotic equipartition property (AEP), the one-shot analysis could, at the very end, be augmented with AEP to yield the same answer. Furthermore, one-shot analysis has been applied to the cases where i.i.d. (independent and identically distributed) and the memoryless assumption is not valid. For example, in a data compression task, we may not have i.i.d. copies of the random variable modeling the source output or in a communication across a noisy channel, the channel may not be memoryless.

The one-shot bounds have been given more recently by Polyanskiy et al. in Ref. [3] and Renner et al. in Ref. [4], [5]. In Ref. [4], [5] Renner et al. introduced the elegant notion of smooth Rényi entropies (defined later) where they also gave one-shot bounds for data compression and randomness extraction (see also Ref. [6]). Another important application of smooth Rényi entropy measure has been the one-shot bounds for the channel capacity given by Renner, Wolf and Wullschleger [7]. Channel coding bounds are also derived in Ref. [8] using a quantity called the smooth 0 -divergence, which is a generalization of Rényi's divergence of order 0 . To the best of our knowledge for the point to point systems Polyanskiy et al's. approach has come out better against the smooth entropy based approach in all the numerical studies done thus far.

The smooth Rényi entropies were extended to the quantum case by Renner and König [9]. There has been a considerable work on the one-shot bounds for the quantum case under various scenarios (see for example Refs. [10], [11], [12], [13], [14], [15] and references therein).

In Ref. [16], Schoenmaker et al introduce the notion of smooth Rényi entropy for the case of stationary ergodic information sources, thereby generalizing previous work which concentrated mainly on i.i.d. information sources. In Ref. [17], Holenstein and Renner give explicit and tight bounds on the smooth entropies of $n$-fold product distributions in terms of the Shannon entropy of a single distribution. Renes and Renner consider the problem of classical data compression in the presence of quantum side information in Ref. [18].

It is worth noting that for the classical case, our 
results (announced in Jan 2012) were the first one-shot multi-party results, i.e., where there are multiple parties at the sender and/or receiver. After our results were were announced on the arxiv, Tan and Kosut also gave non-asymptotic bounds for several multi-party problems following the dispersion based approach [19]. In this paper we concentrate on the distributed encoding (data compression) protocol of correlated information sources and for finding the one-shot transmission rate. We then give one-shot achievable rate pair for the multiple-access channel. In a very remarkable and fundamental paper, Slepian and Wolf showed that there is no loss in the compression efficiency for the distributed encoding as compared to the collaborative encoding [20]. In Ref. [21], Han proves a more general result wherein he gives the asymptotically optimal rate region for distributed source coding in the non i.i.d. regime.

The fundamental contributions by Renner and Wolf [4], [5] have not been extended thus far to the multi-party scenario. The upper bound that Renner and Wolf derive for their data compression comes in the form of two steps. One is the encoding (that can be accomplished by twouniversal hash functions) and second is an elegant step of bootstrapping the first step by encoding a random variable that is in a ball (appropriately defined) around the random variable that we want to compress. Such an approach distributes the error in the two steps in a flexible way and yields an upper bound that is asymptotically tight. To carry over this approach to the Slepian-Wolf protocol would involve proving the existence of more than one random variables that satisfy certain conditions and the authors know of no such proof in the literature.

In this paper, we define a one-shot typical set. We further use this set to give one-shot achievable rates for some multi-party scenarios. Interestingly, our bounds involves smooth Rényi entropy of the order $-\infty$. Rényi entropies are typically defined of order $\alpha \geq 0$ and appropriate limits have to be taken for $\alpha=1$ that corresponds to the Shannon entropy [22]. In the definition of smooth Rényi entropy, we let $\alpha \in[-\infty, \infty]$.

\section{DEFINITION OF VARIOUS ENTROPIES}

We shall assume that all random variables in this paper are discrete and take values over a finite set. Let $X$ be a random variable taking values over alphabet $\mathcal{X}$ with probability mass function (PMF) $P_{X}(x), x \in \mathcal{X}$. Then the Shannon entropy [1] $H(X)$ of $X$ is defined by

$$
H(X):=-\sum_{x \in \mathcal{X}} P_{X}(x) \log \left[P_{X}(x)\right]
$$

where we shall assume that the $\log$ is to the base 2 throughout this paper. The Rényi entropy [22] of $X$ is defined as

$$
H_{\alpha}(X):=\frac{1}{1-\alpha} \log \left[\sum_{x \in \mathcal{X}} P_{X}^{\alpha}(x)\right],
$$

where $\alpha \in[0, \infty]$ and appropriate limits are taken for $\alpha=1$. In particular, for $\alpha=0$, the Rényi entropy is given by

$$
H_{0}(X):=\log \left|\left\{x \in \mathcal{X}: P_{X}(x)>0\right\}\right|
$$

and for $\alpha=\infty$, the Rényi entropy is given by

$$
H_{\infty}(X):=-\log \left[\max _{x \in \mathcal{X}} P_{X}(x)\right] .
$$

Let $X, Y$ be two random variables with joint distribution $P_{X Y}$, the conditional Rényi entropy of order $\alpha$ is defined as

$$
H_{\alpha}(X \mid Y):=\frac{1}{1-\alpha} \log \left[\max _{y \in \mathcal{Y}} \sum_{x \in \mathcal{X}} P_{X \mid Y=y}^{\alpha}(x)\right] .
$$

The $\varepsilon$-smooth conditional Rényi entropy of order $\alpha$ [4] is defined for $\varepsilon \geq 0$ as

$$
\begin{array}{r}
H_{\alpha}^{\varepsilon}(X \mid Y):=\frac{1}{1-\alpha} \log \left[\inf _{\bar{X} \bar{Y}: \operatorname{Pr}[\bar{X} \bar{Y} \neq X Y] \leq \varepsilon} \max _{y \in \mathcal{Y}}\right. \\
\left.\sum_{x \in \mathcal{X}} P_{\bar{X} \mid \bar{Y}=y}^{\alpha}(x)\right] .
\end{array}
$$

Although the Rényi entropy is typically defined for $\alpha \in$ $[0, \infty]$, we let $\alpha \in[-\infty, \infty]$ and, in particular, consider the Rényi entropy of order $\alpha=-\infty$ given by

$$
\begin{gathered}
H_{-\infty}(X \mid Y):=-\log \left[\min _{y \in \mathcal{Y}} \min _{x: P_{X \mid Y=y}(x)>0}\right. \\
\left.P_{X \mid Y=y}(x)\right]
\end{gathered}
$$

and its smooth version for $\varepsilon \geq 0$ as

$$
\begin{aligned}
H_{-\infty}^{\varepsilon}(X \mid Y):=-\log \left[\sup _{\bar{X} \bar{Y}: \operatorname{Pr}[\bar{X} \bar{Y} \neq X Y] \leq \varepsilon} \min _{y \in \mathcal{Y}}\right. & \\
& \left.\min _{x: P_{\bar{X} \mid \bar{Y}=y}(x)>0} P_{\bar{X} \mid \bar{Y}=y}(x)\right] .
\end{aligned}
$$

We shall use $H_{\alpha}^{\varepsilon}(X)$ interchangeably with $H_{\alpha}^{\varepsilon}\left(P_{X}\right)$.

\section{DistRIBUTED ENCODING OF CORRELATED SOURCES}

We describe the task of distributed encoding of correlated sources in this section. Let $(X, Y) \sim P_{X Y}$. Assume that the random variable $X$ is available with Alice at a location $A$ and the random variable $Y$ is available with Bob at a separate location $B$. Both Alice and Bob want to get across the pair $(X, Y)$ to Charlie at location 
$C$ without collaborating with each other within some prescribed error. We state this formally as follows.

Definition 1: For the error $\varepsilon, 0 \leq \varepsilon \leq 1$, let $\Lambda_{\varepsilon}^{P}(\mathcal{X} \times$ $\left.\mathcal{Y} \rightarrow \mathcal{C}_{\mathcal{X}} \times \mathcal{C}_{\mathcal{Y}}\right)$ denote the set $\left(e_{\mathcal{X}}, e_{\mathcal{Y}}, U\right)$ where $U$ is a random variable with range $\mathcal{U}, e_{\mathcal{X}}: \mathcal{X} \times \mathcal{U} \rightarrow \mathcal{C}_{\mathcal{X}}$ and $e_{\mathcal{Y}}: \mathcal{Y} \times \mathcal{U} \rightarrow \mathcal{C}_{\mathcal{Y}}$ such that there exists a decoding function $g: \mathcal{C}_{\mathcal{X}} \times \mathcal{C}_{\mathcal{Y}} \times \mathcal{U} \rightarrow \mathcal{X} \times \mathcal{Y}$ with

$$
\operatorname{Pr}\{(\hat{X}(U), \hat{Y}(U)) \neq(X, Y)\} \leq \varepsilon,
$$

where

$$
(\hat{X}(U), \hat{Y}(U)):=g\left[e_{\mathcal{X}}(X, U), e_{\mathcal{Y}}(Y, U), U\right] .
$$

Occasionally, we shall also use the following

$$
\begin{aligned}
& g_{\mathcal{X}}\left[e_{\mathcal{X}}(X, U), e_{\mathcal{Y}}(Y, U), U\right]:=\hat{X}(U), \\
& g_{\mathcal{Y}}\left[e_{\mathcal{X}}(X, U), e_{\mathcal{Y}}(Y, U), U\right]:=\hat{Y}(U) .
\end{aligned}
$$

The definitions for the encoding lengths for a chosen $\left(e_{\mathcal{X}}, e_{\mathcal{Y}}, U\right) \in \Lambda_{\varepsilon}^{P}$ are given by

$$
\begin{gathered}
\ell_{\mathrm{d}-\mathrm{enc}}^{\varepsilon}(X):=\log \left|\mathcal{C}_{\mathcal{X}}\right|, \\
\ell_{\mathrm{d}-\text { enc }}^{\varepsilon}(Y):=\log \left|\mathcal{C}_{\mathcal{Y}}\right|,
\end{gathered}
$$

where we have written $\Lambda_{\varepsilon}^{P}$ for $\Lambda_{\varepsilon}^{P}\left(\mathcal{X} \times \mathcal{Y} \rightarrow \mathcal{C}_{\mathcal{X}} \times \mathcal{C}_{\mathcal{Y}}\right)$ and we would implicitly assume that $\mathcal{C}_{\mathcal{X}}$ and $\mathcal{C}_{\mathcal{Y}}$ are determined from the same $\left(e_{\mathcal{X}}, e_{\mathcal{Y}}, U\right)$. We also assume that $U$ is independent of $X$ and $Y$.

\section{A. Results}

In this section, we summarize the results and the proofs are given in the next section. We first need the following definition.

Definition 2: For $(X, Y) \sim P_{X Y}$, we define the following sets

$$
\begin{gathered}
\mathcal{A}_{\delta}\left(P_{X}\right):=\left\{x: \Xi_{\min }^{\delta}\left(P_{X}\right) \leq-\log P_{X}(x) \leq\right. \\
\left.\Xi_{\max }^{\delta}\left(P_{X}\right)\right\},
\end{gathered}
$$

where

$$
\begin{aligned}
& \Xi_{\min }^{\delta}\left(P_{X}\right):= H(X)-\left|H(X)-H_{\infty}^{\delta}(X)\right| \\
&-\delta \log |\mathcal{X}| \\
& \Xi_{\max }^{\delta}\left(P_{X}\right):= H(X)+\left|H(X)-H_{-\infty}^{\delta}(X)\right| \\
&+\delta \log |\mathcal{X}| \\
& \mathcal{A}_{\delta}\left(P_{Y}\right):=\left\{y: \Xi_{\min }^{\delta}\left(P_{Y}\right) \leq-\log P_{Y}(y) \leq\right.\left.\Xi_{\max }^{\delta}\left(P_{Y}\right)\right\} \\
& \mathcal{A}_{\delta}\left(P_{X Y}\right):=\left\{(x, y): \Xi_{\min }^{\delta}\left(P_{X Y}\right) \leq-\log P_{X Y}(x, y)\right. \\
& \leq \Xi_{\max }^{\delta}\left(P_{X Y}\right), x \in \mathcal{A}_{\delta}\left(P_{X}\right), y \in \\
&\left.\mathcal{A}_{\delta}\left(P_{Y}\right)\right\}
\end{aligned}
$$

We now give the one-shot bounds for the distributed encoding for correlated sources in the following theorem.

Theorem 1: Let $(X, Y) \sim P_{X Y}$ and $\varepsilon, \varepsilon_{i} \in \mathbb{R}^{+}, i=$ $0,1,2,3$ with $\sum_{i=0}^{3} \varepsilon_{i} \leq \varepsilon$.

Lower bounds: For any distributed encoding protocol, the following lower bounds shall hold

$$
\begin{aligned}
\ell_{\mathrm{d}-\mathrm{enc}}^{\varepsilon}(X) & \geq H_{0}^{\varepsilon}(X \mid Y), \\
\ell_{\mathrm{d}-\mathrm{enc}}^{\varepsilon}(Y) & \geq H_{0}^{\varepsilon}(Y \mid X), \\
\ell_{\mathrm{d}-\mathrm{enc}}^{\varepsilon}(X)+\ell_{\mathrm{d}-\mathrm{enc}}^{\varepsilon}(Y) & \geq H_{0}^{\varepsilon}(X, Y) .
\end{aligned}
$$

Achievability: There exists a distributed encoding protocol for a rate pair $\left(\ell_{\mathrm{d}-\text { enc }}^{\varepsilon}(X), \ell_{\mathrm{d}-\text { enc }}^{\varepsilon}(Y)\right) \quad$ satisfying the following conditions

$$
\begin{aligned}
& \ell_{\mathrm{d}-\mathrm{enc}}^{\varepsilon}(X) \geq \Xi_{\max }^{\delta}\left(P_{X Y}\right)-\Xi_{\min }^{\delta}\left(P_{Y}\right) \\
&-\log \varepsilon_{1}, \\
& \ell_{\mathrm{d}-\mathrm{enc}}^{\varepsilon}(Y) \geq \Xi_{\max }^{\delta}\left(P_{X Y}\right)-\Xi_{\min }^{\delta}\left(P_{X}\right) \\
&-\log \varepsilon_{2}, \\
& \ell_{\mathrm{d}-\mathrm{enc}}^{\varepsilon}(X)+\ell_{\mathrm{d}-\mathrm{enc}}^{\varepsilon}(Y) \geq \Xi_{\max }^{\delta}\left(P_{X Y}\right)-\log \varepsilon_{3},
\end{aligned}
$$

where $\delta$ is chosen such that

$$
\operatorname{Pr}\left\{(X, Y) \notin \mathcal{A}_{\delta}\left(P_{X Y}\right)\right\} \leq \varepsilon_{0} .
$$

Next we show that one-shot rate region in Theorem 1 is asymptotically optimal, i.e., both the lower bounds and the achievable rates (normalized appropriately) are the same when the number of i.i.d. copies of $(X, Y)$ pairs grows unbounded and the errors become arbitrarily small, and that this rate region in the distributed encoding case comes out to be the same as that of Slepian-Wolf [20]. Let

$$
\left(\mathbf{X}_{1}^{n}, \mathbf{Y}_{1}^{n}\right):=\left[\left(X_{1}, Y_{1}\right),\left(X_{2}, Y_{2}\right), \ldots,\left(X_{n}, Y_{n}\right)\right]
$$

where $\left(X_{1}, Y_{1}\right), \ldots\left(X_{n}, Y_{n}\right)$ are $n$ i.i.d. pairs of random variables distributed according to $P_{X Y}$.

Similarly, we can get the asymptotic limit for Theorem 1 which yields the rate-region of Slepian-Wolf [20]. Before we get into that, we first give the definition of rate pair which are achievable asymptotically and this definition is motivated from Ref. [21].

Definition 3: Rate pair $\left(\mathcal{R}_{1}, \mathcal{R}_{2}\right)$ is achievable $\Longleftrightarrow$ There exists a

$$
\left(e_{\mathcal{X}^{n}}, e_{\mathcal{Y}^{n}}, U\right) \in \Lambda_{\varepsilon}^{P_{\mathbf{X}_{1}^{n} \mathbf{Y}_{1}^{n}}}\left(\mathcal{X}^{n} \times \mathcal{Y}^{n} \rightarrow \mathcal{C}_{\mathcal{X}^{n}} \times \mathcal{C}_{\mathcal{Y}^{n}}\right)
$$

and a decoding function $g: \mathcal{C}_{\mathcal{X}^{n}} \times \mathcal{C}_{\mathcal{Y}^{n}} \times \mathcal{U} \rightarrow \mathcal{X}^{n} \times \mathcal{Y}^{n}$ 
such that

$$
\begin{aligned}
\operatorname{Pr}\left\{g\left[e_{\mathcal{X}^{n}}\left(X_{1}^{n}, U\right), e \mathcal{Y}^{n}\left(Y_{1}^{n}, U\right), U\right] \neq\left(X_{1}^{n}, Y_{1}^{n}\right)\right\} & \leq \varepsilon, \\
& \lim _{\varepsilon \rightarrow 0} \limsup _{n \rightarrow \infty} \frac{\ell_{\mathrm{d}-\text { enc }}^{\varepsilon}\left(\mathbf{X}_{1}^{n}\right)}{n} \leq \mathcal{R}_{1} \\
& \lim _{\varepsilon \rightarrow 0} \limsup _{n \rightarrow \infty} \frac{\ell_{\mathrm{d}-\text { enc }}^{\varepsilon}\left(\mathbf{Y}_{1}^{n}\right)}{n} \leq \mathcal{R}_{2} .
\end{aligned}
$$

Lemma 1: The normalized asymptotic limits for the bounds in Theorem 11, are given by

$$
\begin{aligned}
\mathcal{R}_{1} & \geq H\left(X_{1} \mid Y_{1}\right), \\
\mathcal{R}_{2} & \geq H\left(Y_{1} \mid X_{1}\right), \\
\mathcal{R}_{1}+\mathcal{R}_{2} & \geq H\left(X_{1}, Y_{1}\right) .
\end{aligned}
$$

\section{B. Proofs of the results}

Some of the proofs rely upon the two-universal hash functions and we give their definition first (see also Ref. [23] and references therein).

Definition 4: A random function $f: \mathcal{X} \times \mathcal{U} \rightarrow \mathcal{C}$ takes an input $x \in \mathcal{X}$ and generates a uniform random variable $U$ taking values over alphabet $\mathcal{U}$ and outputs $c \in \mathcal{C}$. $f$ is called a two-universal hash function if for any $x \neq x$, $x, x \in \mathcal{X}$, we have

$$
\operatorname{Pr}\{f(x, U)=f(\dot{x}, U)\} \leq \frac{1}{|\mathcal{C}|} .
$$

We prove the following lemma that shall be needed for the probability of error analysis later.

Lemma 2: Let $(X, Y) \sim P_{X Y}$. Define

$$
\mathcal{A}_{\delta}\left(P_{X \mid Y=y}\right):=\left\{x:(x, y) \in \mathcal{A}_{\delta}\left(P_{X Y}\right)\right\} .
$$

For any $\delta \geq 0, y \in \mathcal{A}_{\delta}\left(P_{Y}\right)$, we have

$$
\left|\mathcal{A}_{\delta}\left(P_{X \mid Y=y}\right)\right| \leq 2^{\Xi_{\max }^{\delta}\left(P_{X Y}\right)-\Xi_{\min }^{\delta}\left(P_{Y}\right)} .
$$

Proof: For any $(x, y) \in \mathcal{A}_{\delta}(X, Y)$, we have

$$
P_{X \mid Y=y}(x)=\frac{P_{X Y}(x, y)}{P_{Y}(y)} \geq 2^{-\Xi_{\max }^{\delta}\left(P_{X Y}\right)+\Xi_{\min }^{\delta}\left(P_{Y}\right)} \text {. }
$$

The lemma now follows straightforwardly from

$$
\begin{aligned}
1 & \geq \sum_{x \in \mathcal{A}_{\delta}(X \mid Y=y)} P_{X \mid Y=y}(x) \\
& \geq\left|\mathcal{A}_{\delta}(X \mid Y=y)\right| 2^{-\Xi_{\max }^{\delta}\left(P_{X Y}\right)+\Xi_{\min }^{\delta}\left(P_{Y}\right)},
\end{aligned}
$$

where we have used 35.

The following properties of $H_{-\infty}^{\varepsilon}$ are needed later in the paper.

Lemma 3: The following holds for any $\varepsilon>0$

$$
H_{-\infty}^{\varepsilon}(X) \geq H_{0}^{\varepsilon}(X) \text {. }
$$

Proof: For all $\delta>0$, there exists a random variable $\bar{X}$ with $\operatorname{Pr}\{X \neq \bar{X}\} \leq \varepsilon$ such that

$$
H_{-\infty}^{\varepsilon}(X) \geq H_{-\infty}(\bar{X})-\delta \text {. }
$$

Now the following inequalities hold

$$
\begin{aligned}
H_{-\infty}^{\varepsilon}(X) & \geq H_{-\infty}(\bar{X})-\delta \\
& \geq H_{0}(\bar{X})-\delta \\
& \geq H_{0}^{\varepsilon}(X)-\delta .
\end{aligned}
$$

Since this is true for any $\delta>0$, the result follows.

Lemma 4: If $X_{1}^{n}=\left[X_{1}, \ldots, X_{n}\right]$ be $n$ i.i.d. random variables distributed according to $P_{X}$, then

$$
\lim _{\varepsilon \rightarrow 0} \lim _{n \rightarrow \infty} \frac{H_{-\infty}^{\varepsilon}\left(X_{1}^{n}\right)}{n}=H\left(X_{1}\right) .
$$

Proof: The typical set, $\mathcal{T}_{\varepsilon}^{(n)} \subseteq \mathcal{X}^{n}, \varepsilon \geq 0$ (see Ref. [2] for details) is defined as

$$
\mathcal{T}_{\varepsilon}^{(n)}=\left\{x^{n}:\left|-\frac{1}{n} \log P\left(x_{1}^{n}\right)-H\left(X_{1}\right)\right| \leq \varepsilon\right\} .
$$

We now define a random variable $Z$ as

$$
Z= \begin{cases}X_{1}^{n} & \text { if } X_{1}^{n} \in \mathcal{T}_{\varepsilon}^{(n)} \\ W & \text { if } X_{1}^{n} \notin \mathcal{T}_{\varepsilon}^{(n)}\end{cases}
$$

where $W$ is uniformly chosen at random from the set $\mathcal{T}_{\varepsilon}^{(n)}$. We now have

$$
\begin{aligned}
\operatorname{Pr}\left\{Z \neq X_{1}^{n}\right\} & =\operatorname{Pr}\left\{X_{1}^{n} \notin \mathcal{T}_{\varepsilon}^{(n)}\right\} \\
& \leq \varepsilon
\end{aligned}
$$

where we have assumed that $n$ is large enough so that (47) is satisfied. To get a bound on $\min _{z} \operatorname{Pr}\{Z=z\}$, we note that for any $x_{1}^{n} \in \mathcal{T}_{\varepsilon}^{(n)}, \operatorname{Pr}\left\{X_{1}^{n}=x_{1}^{n}\right\}$ $\geq 2^{-n\left(H\left(X_{1}\right)+\varepsilon\right)}$. Now using the definition of $H_{-\infty}^{\varepsilon}(X)$ and $\operatorname{Pr}\left\{Z \neq X_{1}^{n}\right\} \leq \varepsilon$, we have $H_{-\infty}^{\varepsilon}\left(X_{1}^{n}\right) \leq H_{-\infty}(Z)$, and hence,

$$
\frac{H_{-\infty}^{\varepsilon}\left(X_{1}^{n}\right)}{n} \leq \frac{H_{-\infty}(Z)}{n} \leq H\left(X_{1}\right)+\varepsilon,
$$

or

$$
\lim _{\varepsilon \rightarrow 0} \lim _{n \rightarrow \infty} \frac{H_{-\infty}\left(X_{1}^{n}\right)}{n} \leq H\left(X_{1}\right) .
$$

We now use Lemma 3 to get $H_{-\infty}^{\varepsilon}\left(X_{1}^{n}\right) \geq H_{0}^{\varepsilon}\left(X_{1}^{n}\right)$ and hence,

$$
\lim _{\varepsilon \rightarrow 0} \lim _{n \rightarrow \infty} \frac{H_{-\infty}^{\varepsilon}\left(X_{1}^{n}\right)}{n} \geq \lim _{\varepsilon \rightarrow 0} \lim _{n \rightarrow \infty} \frac{H_{0}^{\varepsilon}\left(X_{1}^{n}\right)}{n}=H\left(X_{1}\right),
$$

where the equality in (50) follows from Ref. [5]. Thus, from (49) and (50), the lemma is proved. 


\section{Proof of the lower bound in Theorem $\square$}

Let $\left(e_{\mathcal{X}}, e_{\mathcal{Y}}, R\right) \in \Lambda_{\varepsilon}^{P}\left(\mathcal{X} \times \mathcal{Y} \rightarrow \mathcal{C}_{\mathcal{X}} \times \mathcal{C}_{\mathcal{Y}}\right)$. Then there must exist a realization $R=r$ such that the error bound in (9) is met. We first prove 201.

$$
\begin{aligned}
\ell_{\mathrm{d}-\mathrm{enc}}^{\varepsilon}(X) & \stackrel{a}{=} H_{0}\left[e_{\mathcal{X}}(X, r)\right] \\
& \stackrel{b}{\geq} H_{0}\left[e_{\mathcal{X}}(X, r) \mid Y\right] \\
& \stackrel{c}{=} H_{0}\left[e_{\mathcal{X}}(X, r), e_{\mathcal{Y}}(Y, r) \mid Y\right] \\
& \stackrel{d}{\geq} H_{0}\left\{g_{\mathcal{X}}\left[e_{\mathcal{X}}(X, r), e_{\mathcal{Y}}(Y, r), r\right] \mid Y\right\} \\
& =H_{0}[\hat{X}(r) \mid Y] \\
& \stackrel{e}{\geq} H_{0}^{\varepsilon}(X \mid Y),
\end{aligned}
$$

where $a$ follows from the definition of $\ell_{\mathrm{d}-\mathrm{enc}}^{\varepsilon}(X), b$ follows since conditioning reduces $H_{0}, c$ is an identity that is easily derived from the definition of $H_{0}, d$ follows since taking a function reduces $H_{0}$, and $e$ follows since $\operatorname{Pr}\{\hat{X}(r) \neq X\} \leq \varepsilon$ and from (6).

The proof for 21) is similar and is omitted. We now prove 22.

$$
\begin{aligned}
\ell_{\mathrm{d}-\mathrm{enc}}^{\varepsilon}(X)+\ell_{\mathrm{d}-\mathrm{enc}}^{\varepsilon}(Y) & =H_{0}\left[e_{\mathcal{X}}(X, r)\right]+H_{0}\left[e_{\mathcal{Y}}(Y, r)\right] \\
& \geq H_{0}\left[e_{\mathcal{X}}(X, r), e_{\mathcal{Y}}(Y, r)\right] \\
& \geq H_{0}\left\{g\left[e_{\mathcal{X}}(X, r), e_{\mathcal{Y}}(Y, r), r\right]\right\} \\
& =H_{0}[\hat{X}(r), \hat{Y}(r)] \\
& \geq H_{0}^{\varepsilon}(X, Y),
\end{aligned}
$$

where $a$ follows from the sub-additivity of the Rényi entropy.

\section{Proof of achievability in Theorem 1$]$}

We show the existence of a protocol for distributed encoding (again based on two-universal hash functions) and is given by the following steps.

1) Let $e_{\mathcal{X}}: \mathcal{X} \times \mathcal{U} \rightarrow \mathcal{C}_{\mathcal{X}}, e_{\mathcal{Y}}: \mathcal{Y} \times \mathcal{U} \rightarrow \mathcal{C}_{\mathcal{Y}}$ be two-universal hash functions and let $\mathcal{C}_{\mathcal{X}}=$ $\left\{1,2, \cdots, 2^{\ell_{\mathcal{X}}}\right\}$ and $\mathcal{C}_{\mathcal{Y}}=\left\{1,2, \cdots, 2^{\ell \mathcal{Y}}\right\}$. Alice takes $x \in \mathcal{X}$, generates $U$ (known both to Bob and Charlie), and sends $i=e_{\mathcal{X}}(x, U)$ to Charlie. Similarly, Bob takes $y \in \mathcal{Y}$ and sends $j=e_{\mathcal{Y}}(y, U)$ to Charlie.

2) Charlie passes the received indices $(i, j)$ to the decoder $g: \mathcal{C}_{\mathcal{X}} \times \mathcal{C}_{\mathcal{Y}} \times \mathcal{U} \rightarrow \mathcal{X} \times \mathcal{Y}$ that outputs $(\hat{x}, \hat{y})=(x, y)$ if there is only one pair $(x, y)$ such that $e_{\mathcal{X}}(x, U)=i, e_{\mathcal{Y}}(y, U)=j$ and $(x, y) \in$ $\mathcal{A}_{\delta}\left(P_{X Y}\right)$. In all other cases, it declares an error.

Let $\varepsilon_{0}, \varepsilon_{1}, \varepsilon_{2}, \varepsilon_{3} \in \mathbb{R}^{+}$and $\sum_{k=0}^{3} \varepsilon_{k} \leq \varepsilon$. The probability of error of the above protocol is computed by defining the following events.

$$
\begin{aligned}
& E_{0}:=\left\{(X, Y) \notin \mathcal{A}_{\delta}\left(P_{X Y}\right)\right\}, \\
& E_{1}:=\left\{\exists \dot{x} \neq X: e_{\mathcal{X}}(\dot{x}, U)=e_{\mathcal{X}}(X, U),(\dot{x}, Y)\right.\left.\in \mathcal{A}_{\delta}\left(P_{X Y}\right)\right\} . \\
& E_{2}:=\left\{\exists \dot{y} \neq Y: e_{\mathcal{Y}}(\dot{y}, U)=e_{\mathcal{Y}}(Y, U),(X, \dot{y})\right. \\
&\left.\in \mathcal{A}_{\delta}\left(P_{X Y}\right)\right\} . \\
& E_{12}:=\left\{\exists(\dot{x}, \dot{y}):(\dot{x}, \dot{y}) \neq(X, Y), e_{\mathcal{X}}(\dot{x}, U)=\right. \\
& e_{\mathcal{X}}(X, U), e_{\mathcal{Y}}(\dot{y}, U)=e_{\mathcal{Y}}(Y, U),(\dot{x}, \dot{y}) \\
&\left.\in \mathcal{A}_{\delta}\left(P_{X Y}\right)\right\} .
\end{aligned}
$$

The probability of error is given by the probability of the union of these events and we upper bound that by the union bound as

$$
\begin{aligned}
P_{e} & =\operatorname{Pr}\left\{E_{0} \cup E_{1} \cup E_{2} \cup E_{12}\right\} \\
& \leq \operatorname{Pr}\left\{E_{0}\right\}+\operatorname{Pr}\left\{E_{1}\right\}+\operatorname{Pr}\left\{E_{2}\right\}+\operatorname{Pr}\left\{E_{12}\right\} .
\end{aligned}
$$

For further details on the calculation of $P_{e}$ and the proofs of (23), 24) and (25) see Ref. [24].

\section{E. Proof of Lemma 1}

The proof of Lemma 1 follow straightforwardly if we could invoke Lemma 4 , which we can if we can show that $\delta$ is not zero for large enough $n$. It is here that we shall need the following remarkable result by Holenstein and Renner [17] giving explicit bounds.

Theorem 2: (Holenstein and Renner [17]) Let $P_{\mathbf{X}_{1}^{n} \mathbf{Y}_{1}^{n}}:=P_{X_{1} Y_{1}} \cdots P_{X_{n} Y_{n}}$ be a probability distribution over $\mathcal{X}^{n} \times \mathcal{Y}^{n}$. Then, for any $\delta \in[0, \log |\mathcal{X}|]$ and $\mathbf{x}, \mathbf{y}$ chosen according to $P_{\mathbf{X}_{1}^{n}} \mathbf{Y}_{1}^{n}$

$$
\operatorname{Pr}_{\mathbf{x}, \mathbf{y}}\left[-\log \left(P_{\mathbf{X}_{1}^{n} \mid \mathbf{Y}_{1}^{n}}(\mathbf{x}, \mathbf{y})\right) \geq H\left(\mathbf{X}_{1}^{n} \mid \mathbf{Y}_{1}^{n}\right)+n \delta\right] \leq \varepsilon
$$

and, similarly

$$
\underset{\mathbf{x}, \mathbf{y}}{\operatorname{Pr}}\left[-\log \left(P_{\mathbf{X}_{1}^{n} \mid \mathbf{Y}_{1}^{n}}(\mathbf{x}, \mathbf{y})\right) \leq H\left(\mathbf{X}_{1}^{n} \mid \mathbf{Y}_{1}^{n}\right)-n \delta\right] \leq \varepsilon
$$

where $\varepsilon=2^{-\frac{n \delta^{2}}{2 \log ^{2}(|\mathcal{X}|+3)}}$.

Let us now assume that we want to ensure that for $\delta=\varepsilon_{0}$,

$$
\operatorname{Pr}\left\{\mathbf{X}_{1}^{n} \notin A_{\delta}\left(P_{\mathbf{X}_{1}^{n}}\right)\right\} \leq \varepsilon_{0} .
$$

Recall that $X_{1}, X_{2}, \ldots$ are i.i.d. and distributed according to $P_{X}$. Then it is sufficient to ensure that

$$
\begin{aligned}
& \operatorname{Pr}_{\mathbf{x}}\left[-\log \left(P_{\mathbf{X}_{1}^{n}}(\mathbf{x})\right) \geq n H\left(X_{1}\right)+n \delta \log |\mathcal{X}|\right] \leq \frac{\varepsilon_{0}}{2}, \\
& \operatorname{Pr}_{\mathbf{x}}\left[-\log \left(P_{\mathbf{X}_{1}^{n}}(\mathbf{x})\right) \leq n H\left(X_{1}\right)-n \delta \log |\mathcal{X}|\right] \leq \frac{\varepsilon_{0}}{2},
\end{aligned}
$$

where we have taken out the term inside $|\cdot|$ to get upper bounds to the probabilities. Now invoking the result by 
Holenstein and Renner [17], if we choose $\delta=\varepsilon_{0}$, then for all $n \geq n_{0}\left(\varepsilon_{0}\right)$, where

$$
n_{0}\left(\varepsilon_{0}\right):=\sqrt{-\frac{2 \log \left(\varepsilon_{0} / 2\right) \log ^{2}(|\mathcal{X}|+3)}{\log ^{2}(|\mathcal{X}|) \varepsilon_{0}^{2}}},
$$

the error bound in (57) is met. Note that we could choose $\delta=0$ for $n<n_{0}\left(\varepsilon_{0}\right)$. Hence, both the sequences $\left\{H_{-\infty}^{\delta}\left(X_{1}^{n}\right) / n\right\}$ and $\left\{H_{-\infty}^{\varepsilon_{0}}\left(X_{1}^{n}\right) / n\right\}$ are equal $\forall n \geq$ $n_{0}\left(\varepsilon_{0}\right)$. Hence,

$$
\begin{aligned}
\lim _{\varepsilon \rightarrow 0} \lim _{n \rightarrow \infty} \frac{H_{-\infty}^{\delta}\left(X_{1}^{n}\right)}{n} & =\lim _{\varepsilon \rightarrow 0} \lim _{n \rightarrow \infty} \frac{H_{-\infty}^{\varepsilon_{0}}\left(X_{1}^{n}\right)}{n} \\
& =\lim _{\varepsilon_{0} \rightarrow 0} \lim _{n \rightarrow \infty} \frac{H_{-\infty}^{\varepsilon_{0}}\left(X_{1}^{n}\right)}{n} .
\end{aligned}
$$

Now Lemma 4 can be invoked to yield the desired answer. It is straightforward to extend the above argument for the i.i.d. sequence of a pair of random variables $(X, Y)$.

Since, the bounds derived in the achievability part of Theorem 1 are true for any $n$, therefore using Definition 10 and the above discussion we get the following bounds in the asymptotic regime for any achievable rate pair $\left(\mathcal{R}_{1}, \mathcal{R}_{2}\right)$

$$
\begin{gathered}
\mathcal{R}_{1} \geq \lim _{\varepsilon \rightarrow 0} \limsup _{n \rightarrow \infty} \frac{\ell_{\mathrm{d}-\mathrm{enc}}^{\varepsilon}\left(\mathbf{X}_{1}^{n}\right)}{n} \geq H\left(X_{1} \mid Y_{1}\right), \\
\mathcal{R}_{2} \geq \lim _{\varepsilon \rightarrow 0} \limsup _{n \rightarrow \infty} \frac{\ell_{\mathrm{d}-\mathrm{enc}}^{\varepsilon}\left(\mathbf{Y}_{1}^{n}\right)}{n} \geq H\left(Y_{1} \mid X_{1}\right), \\
\mathcal{R}_{1}+\mathcal{R}_{2} \geq \lim _{\varepsilon \rightarrow 0} \limsup _{n \rightarrow \infty}\left[\frac{\ell_{\mathrm{d}-\text { enc }}^{\varepsilon}\left(\mathbf{X}_{1}^{n}\right)+\ell_{\mathrm{d}-\text { enc }}^{\varepsilon}\left(\mathbf{Y}_{1}^{n}\right)}{n}\right] \\
\geq H\left(X_{1}, Y_{1}\right),
\end{gathered}
$$

where $a, b$ follow from the Definition 10 and $c$ follows because

$$
\begin{aligned}
& \lim _{\varepsilon \rightarrow 0} \limsup _{n \rightarrow \infty} \frac{\ell_{\mathrm{d}-\text { enc }}^{\varepsilon}\left(\mathbf{X}_{1}^{n}\right)}{n}+\lim _{\varepsilon \rightarrow 0} \limsup _{n \rightarrow \infty} \frac{\ell_{\mathrm{d}-\text { enc }}^{\varepsilon}\left(\mathbf{Y}_{1}^{n}\right)}{n} \geq \\
& \lim _{\varepsilon \rightarrow 0} \limsup _{n \rightarrow \infty}\left[\frac{\ell_{\mathrm{d}-\text { enc }}^{\varepsilon}\left(\mathbf{X}_{1}^{n}\right)+\ell_{\mathrm{d}-\text { enc }}^{\varepsilon}\left(\mathbf{Y}_{1}^{n}\right)}{n}\right] .
\end{aligned}
$$

\section{Multiple Access Channel}

Definition 5: A two user discrete multiple-access channel consists of three alphabets $\mathcal{X}, \mathcal{Y}, \mathcal{Z}$ and a probability transition matrix $P_{Z \mid X, Y}$.

Definition 6: A $\left(2^{C_{1}^{\varepsilon}\left(\mathcal{M}^{(1)}\right)}, 2^{C_{2}^{\varepsilon}\left(\mathcal{M}^{(1)}\right)}\right)$ one-shot code for the two user multiple-access channel consists of two message sets $\mathcal{M}^{(1)}=\left[1: 2^{C_{1}^{\varepsilon}\left(\mathcal{M}^{(1)}\right)}\right], \mathcal{M}^{(2)}=[1:$
$\left.2^{C_{2}^{\varepsilon}\left(\mathcal{M}^{(2)}\right)}\right]$ and a random variable $U$ with range $\mathcal{U}$, two encoding functions,

$$
\begin{aligned}
& e_{\mathcal{M}^{(1)}}: \mathcal{M}^{(1)} \rightarrow \mathcal{X}, \\
& e_{\mathcal{M}^{(2)}}: \mathcal{M}^{(2)} \rightarrow \mathcal{Y},
\end{aligned}
$$

and a decoding function

$$
g: \mathcal{Z} \rightarrow \mathcal{M}^{(1)} \times \mathcal{M}^{(2)} .
$$

We will call

$$
C_{1}^{\varepsilon}\left(\mathcal{M}^{(1)}\right)=\log \left|\mathcal{M}^{(1)}\right|, \quad C_{2}^{\varepsilon}\left(\mathcal{M}^{(1)}\right)=\log \left|\mathcal{M}^{(2)}\right|
$$

the coding rates of the encoders 1 and 2 .

Definition 7: An $\varepsilon$ one-shot rate pair, denoted by $\left(\mathcal{R}_{1}^{\varepsilon}, \mathcal{R}_{2}^{\varepsilon}\right)$, is said to be achievable for the two user multiple-access channel if and only if there exists a code $\left(2^{C_{1}^{\varepsilon}\left(\mathcal{M}^{(1)}\right)}, 2^{C_{2}^{\varepsilon}\left(\mathcal{M}^{(1)}\right)}\right)$ such that

$$
\begin{aligned}
\operatorname{Pr}\{g(Z) & \left.\neq\left(M_{1}, M_{2}\right)\right\} \leq \varepsilon, \\
\mathcal{R}_{1}^{\varepsilon} & \leq C_{1}^{\varepsilon}\left(\mathcal{M}^{(1)}\right), \\
\mathcal{R}_{2}^{\varepsilon} & \leq C_{1}^{\varepsilon}\left(\mathcal{M}^{(2)}\right) .
\end{aligned}
$$

Definition 8: A $\left(2^{C_{1}^{\varepsilon}\left(\mathcal{M}_{n}^{(1)}\right)}, 2^{C_{2}^{\varepsilon}\left(\mathcal{M}_{n}^{(1)}\right)}\right) n$-shot code for the two user multiple-access channel consists of two message sets $\mathcal{M}_{n}^{(1)}=\left[1: 2^{C_{1}^{\varepsilon}\left(\mathcal{M}_{n}^{(1)}\right)}\right], \mathcal{M}_{n}^{(2)}=[1$ : $\left.2^{C_{2}^{\varepsilon}\left(\mathcal{M}_{n}^{(2)}\right)}\right]$ and a random variable $U$ with range $\mathcal{U}$, two encoding functions,

$$
\begin{aligned}
& e_{\mathcal{M}_{n}^{(1)}}: \mathcal{M}_{n}^{(1)} \rightarrow \mathcal{X}^{n}, \\
& e_{\mathcal{M}_{n}^{(2)}}: \mathcal{M}_{n}^{(2)} \rightarrow \mathcal{Y}^{n},
\end{aligned}
$$

and a decoding function

$$
g: \mathcal{Z}^{n} \rightarrow \mathcal{M}_{n}^{(1)} \times \mathcal{M}_{n}^{(2)} .
$$

where $\mathcal{X}^{n}, \mathcal{Y}^{n}$ and $\mathcal{Z}^{n}$ denote the $\mathrm{n}$-fold Cartesian product of $\mathcal{X}, \mathcal{Y}$ and $\mathcal{Z}$. We will call

$$
\begin{aligned}
& \frac{C_{1}^{\varepsilon}\left(\mathcal{M}_{n}^{(1)}\right)}{n}=\frac{\log \left|\mathcal{M}_{n}^{(1)}\right|}{n}, \\
& \frac{C_{2}^{\varepsilon}\left(\mathcal{M}_{n}^{(2)}\right)}{n}=\frac{\log \left|\mathcal{M}_{n}^{(2)}\right|}{n}
\end{aligned}
$$

as the coding rate for encoders 1 and 2 .

Definition 9: Let $x^{n} \in \mathcal{X}^{n}$ and $y^{n} \in \mathcal{Y}^{n}$ be two $n$ length input sequences of a two user multiple-access channel and let $z^{n} \in \mathcal{Z}^{n}$ be the output sequence of the channel. We call the channel to be memoryless if

$$
P_{Z^{n} \mid X^{n}, Y^{n}}\left(z^{n} \mid x^{n}, y^{n}\right)=\prod_{i=1}^{n} P_{Z \mid X, Y}\left(z_{i} \mid x_{i}, y_{i}\right) .
$$

We now give the definition of rate pair which is achievable asymptotically for the two user multiple access channel. 
Definition 10: Rate pair $\left(\mathcal{R}_{1}, \mathcal{R}_{2}\right)$ is achievable $\Longleftrightarrow$ There exists a triplet $\left(e_{\mathcal{M}_{n}^{(1)}}, e_{\mathcal{M}_{n}^{(2)}}, g\right)$, such that

$$
\begin{aligned}
&\left.\operatorname{Pr}\left\{g\left(Z^{n}, U\right)\right\} \neq\left(M_{1}, M_{2}\right)\right\} \leq \varepsilon, \\
& \lim _{\varepsilon \rightarrow 0} \liminf _{n \rightarrow \infty} \frac{C_{1}^{\varepsilon}\left(\mathcal{M}_{n}^{(1)}\right)}{n} \geq \mathcal{R}_{1}, \\
& \lim _{\varepsilon \rightarrow 0} \liminf _{n \rightarrow \infty} \frac{C_{2}^{\varepsilon}\left(\mathcal{M}_{n}^{(2)}\right)}{n} \geq \mathcal{R}_{2} .
\end{aligned}
$$

Definition 11: For $(X, Y, Z) \sim P_{X Y Z}$, we define the following sets where

$$
\begin{aligned}
\mathcal{A}_{\delta}\left(P_{X Y Z}\right):= & \left\{(x, y, z): \Xi_{\min }^{\delta}\left(P_{X Y Z}\right) \leq-\log P_{X Y Z}(x, y, z)\right. \\
& \leq \Xi_{\max }^{\delta}\left(P_{X Y Z}\right), x \in \mathcal{A}_{\delta}\left(P_{X Y}\right) \cap \mathcal{A}_{\delta}\left(P_{X Z}\right), \\
z & \in \mathcal{A}_{\delta}\left(P_{X Z}\right) \cap \mathcal{A}_{\delta}\left(P_{Y Z}\right), y \in \mathcal{A}_{\delta}\left(P_{X Y}\right) \cap \\
& \left.\mathcal{A}_{\delta}\left(P_{Y Z}\right)\right\},
\end{aligned}
$$

where

$$
\begin{aligned}
\Xi_{\min }^{\delta}\left(P_{X Y Z}\right):= & H(X Y Z)-\left|H(X Y Z)-H_{\infty}^{\delta}(X Y Z)\right| \\
& -\delta(\log |\mathcal{X}|+\log |\mathcal{Y}|+\log |\mathcal{Z}|), \\
\Xi_{\max }^{\delta}\left(P_{X Y Z}\right):= & H(X Y Z)+\left|H(X Y Z)-H_{-\infty}^{\delta}(X Y Z)\right| \\
& +\delta(\log |\mathcal{X}|+\log |\mathcal{Y}|+\log |\mathcal{Z}|),
\end{aligned}
$$

and $\mathcal{A}_{\delta}\left(P_{X Y}\right), \mathcal{A}_{\delta}\left(P_{X Z}\right)$ and $\mathcal{A}_{\delta}\left(P_{Y Z}\right)$ are defined in similar way as 19].

We give one-shot bounds for the transmission rates of the user 1 and user 2 for the multiple-access channel in the following theorem.

Theorem 3: Let $(X, Y, Z) \sim P_{X Y Z}$ and $\varepsilon, \varepsilon_{i} \in \mathbb{R}^{+}$, $i=0,1,2,3$ with $\sum_{i=0}^{3} \varepsilon_{i} \leq \varepsilon$.

Achievability: There exists a one-shot communication protocol for the multiple-access channel for a rate pair $\left(C_{1}^{\varepsilon}, C_{2}^{\varepsilon}\right)$ satisfying the following conditions

$$
\begin{aligned}
& C_{1}^{\varepsilon} \leq \Xi_{\min }^{\delta}\left(P_{X}\right)+\Xi_{\min }^{\delta}\left(P_{Y Z}\right)-\Xi_{\max }^{\delta}\left(P_{X Y Z}\right) \\
&+\log \left(\varepsilon_{1}\right), \\
& C_{2}^{\varepsilon} \leq \Xi_{\min }^{\delta}\left(P_{Y}\right)+\Xi_{\min }^{\delta}\left(P_{X Z}\right)-\Xi_{\max }^{\delta}\left(P_{X Y Z}\right) \\
&+\log \left(\varepsilon_{2}\right) \\
& C_{1}^{\varepsilon}+C_{2}^{\varepsilon} \leq \Xi_{\min }^{\delta}\left(P_{X}\right)+\Xi_{\min }^{\delta}\left(P_{Y}\right)+\Xi_{\min }^{\delta}\left(P_{Z}\right)- \\
& \Xi_{\max }^{\delta}\left(P_{X Y Z}\right)+\log \left(\varepsilon_{3}\right) .
\end{aligned}
$$

where $\delta$ is chosen such that

$$
\operatorname{Pr}\left\{(X, Y, Z) \notin \mathcal{A}_{\delta}\left(P_{X Y Z}\right)\right\} \leq \varepsilon_{0} .
$$

Proof:
We show the existence of a one-shot communication protocol for the multiple-access channel (based on twouniversal hash functions) and is given by the following steps. Fix a $P_{X Y}=P_{X} P_{Y}$.

1) Codebook generation. Randomly and independently generate $2^{C_{1}^{\varepsilon}}$ codewords $x\left(m_{1}\right), m_{1} \in[1$ : $\left.2^{C_{1}^{\varepsilon}}\right]$, each according to $P_{X}$. Similarly generate $2^{C_{2}^{\varepsilon}}$ codewords $x\left(m_{2}\right), m_{2} \in\left[1: 2^{C_{2}^{\varepsilon}}\right]$, each according to $P_{Y}$. These codewords form the codebook, which is revealed to the senders and the receiver.

2) Encoding. To send a message $m_{1}$, sender 1 sends the codeword $X\left(m_{1}\right)$. Similarly, to send $m_{2}$ sender 2 sends $Y\left(m_{2}\right)$.

3) Decoding. The receiver $Z$ chooses the pair $\left(m_{1}, m_{2}\right)$ such that

$$
\left(x\left(m_{1}\right), y\left(m_{2}\right), z\right) \in \mathcal{A}_{\delta}\left(P_{X Y Z}\right)
$$

if such a pair exists and is unique; otherwise, an error is declared.

4) Probability of error analysis. By the symmetry of the random code construction, the conditional probability of error does not depend on which pair of messages is sent. Thus, without loss of generality, we assume that the message pair $\left(m_{1}, m_{2}\right)$ was sent.

Let $\varepsilon_{0}, \varepsilon_{1}, \varepsilon_{2}, \varepsilon_{3} \in \mathbb{R}^{+}$and $\sum_{k=0}^{3} \varepsilon_{k} \leq \varepsilon$. The probability of error of the above protocol is computed by defining the following events.

$$
\begin{aligned}
E_{0}:= & \left\{\left(X\left(m_{1}\right), Y\left(m_{2}\right), Z\right) \notin \mathcal{A}_{\delta}\left(P_{X Y Z}\right)\right\}, \\
E_{m_{1}, m_{2}}:= & \left\{\exists\left(m_{1}, m_{2}\right) \neq\left(m_{1}, m_{2}\right):\left(X\left(m_{1}\right),\right.\right. \\
& \left.\left.Y\left(m_{2}\right), Z\right) \in \mathcal{A}_{\delta}\left(P_{X Y Z}\right)\right\} .
\end{aligned}
$$

The probability of error is given by the probability of the union of these events and we upper bound that by the union bound as

$$
\begin{aligned}
P_{e}= & \operatorname{Pr}\left\{E_{0} \bigcup \cup_{\left(m_{1}^{\prime}, m_{2}\right) \neq\left(m_{1}, m_{2}\right)} E_{m_{1}, m_{2}}\right\} \\
\leq & \operatorname{Pr}\left\{E_{0}\right\}+\sum_{m_{1}^{\prime} \neq m_{1}, m_{2}=m_{2}} \operatorname{Pr}\left\{E_{m_{1}, m_{2}}\right\} \\
& +\sum_{m_{1}^{\prime}=m_{1}, m_{2}^{\prime} \neq m_{2}} \operatorname{Pr}\left\{E_{m_{1}, m_{2}}\right\} \\
& +\sum_{m_{1}^{\prime} \neq m_{1}, m_{2}^{\prime} \neq m_{2}} \operatorname{Pr}\left\{E_{m_{1}^{\prime}, m_{2}}\right\} .
\end{aligned}
$$

For any $\varepsilon_{0} \geq 0$, we could choose $\delta \geq 0$ such that $\operatorname{Pr}\left\{E_{0}\right\} \leq \varepsilon_{0}$. 
For $m_{1} \neq m_{1}$, we have

$$
\begin{aligned}
\operatorname{Pr}\left\{E_{m_{1}, m_{2}}\right\} & =\operatorname{Pr}\left\{\left(X\left(m_{1}\right), Y\left(m_{2}\right), Z\right) \in \mathcal{A}_{\delta}\left(P_{X Y Z}\right)\right\} \\
& =\sum_{(x, y, z) \in \mathcal{A}_{\delta}\left(P_{X Y Z}\right)} \operatorname{Pr}(x) \operatorname{Pr}(y, z) \\
& \leq\left|\mathcal{A}_{\delta}\left(P_{X Y Z}\right)\right| 2^{-\left(\Xi_{\min }^{\delta}\left(P_{X}\right)+\Xi_{\min }^{\delta}\left(P_{Y Z}\right)\right)} \\
& \leq 2^{-\left(\Xi_{\min }^{\delta}\left(P_{X}\right)+\Xi_{\min }^{\delta}\left(P_{Y Z}\right)-\Xi_{\max }^{\delta}\left(P_{X Y Z}\right)\right)},
\end{aligned}
$$

where $a$ and $b$ follows from the properties of $\mathcal{A}_{\delta}\left(P_{X Y Z}\right)$. Similarly for $m_{2} \neq m_{2}$, we have

$$
\begin{aligned}
& \operatorname{Pr}\left\{E_{m_{1}, m_{2}}\right\} \leq 2^{-\left(\Xi_{\min }^{\delta}\left(P_{Y}\right)+\Xi_{\min }^{\delta}\left(P_{X Z}\right)-\Xi_{\max }^{\delta}\left(P_{X Y Z}\right)\right)} \\
& \text { and for } m_{1} \neq m_{1}, m_{2}^{\prime} \neq m_{2}, \\
& \operatorname{Pr}\left\{E_{m_{1}}, m_{2}^{\prime}\right\} \leq 2^{-\left(\Xi_{\min }^{\delta}\left(P_{X}\right)+\Xi_{\min }^{\delta}\left(P_{Y}\right)+\Xi_{\min }^{\delta}\left(P_{Z}\right)-\Xi_{\max }^{\delta}\left(P_{X Y Z}\right)\right)} \\
& \text { Thus, } \\
& P_{e} \leq \varepsilon_{0}+2^{C_{1}^{\varepsilon}} 2^{-\left(\Xi_{\min }^{\delta}\left(P_{X}\right)+\Xi_{\min }^{\delta}\left(P_{Y Z}\right)-\Xi_{\max }^{\delta}\left(P_{X Y Z}\right)\right)} \\
& \quad+2^{C_{2}^{\varepsilon}} 2^{-\left(\Xi_{\min }^{\delta}\left(P_{Y}\right)+\Xi_{\min }^{\delta}\left(P_{X Z}\right)-\Xi_{\max }^{\delta}\left(P_{X Y Z}\right)\right)}+2^{C_{1}^{\varepsilon}+C_{2}^{\varepsilon}} \\
& \quad 2^{-\left(\Xi_{\min }^{\delta}\left(P_{X}\right)+\Xi_{\min }^{\delta}\left(P_{Y}\right)+\Xi_{\min }^{\delta}\left(P_{Z}\right)-\Xi_{\max }^{\delta}\left(P_{X Y Z}\right)\right)} .
\end{aligned}
$$

Hence, $\sum_{m_{1} \neq m_{1}, m_{2}=m_{2}} \operatorname{Pr}\left\{E_{m_{1}, m_{2}}\right\} \leq \varepsilon_{1}$ if $C_{1}^{\varepsilon} \leq \Xi_{\min }^{\delta}\left(P_{X}\right)+\Xi_{\min }^{\delta}\left(P_{Y Z}\right)-\Xi_{\max }^{\delta}\left(P_{X Y Z}\right)+$ $\log \left(\varepsilon_{1}\right)$. Similarly, we could show that $\sum_{m_{1}=m_{1}, m_{2} \neq m_{2}} \operatorname{Pr}\left\{E_{m_{1}, m_{2}}\right\} \quad \leq \quad \varepsilon_{2}$ if $C_{2}^{\varepsilon} \leq \Xi_{\min }^{\delta}\left(P_{Y}\right)+\Xi_{\min }^{\delta}\left(P_{X Z}\right)-\Xi_{\max }^{\delta}\left(P_{X Y Z}\right)+\log \left(\varepsilon_{2}\right)$ and $\sum_{m_{1} \neq m_{1}, m_{2} \neq m_{2}} \operatorname{Pr}\left\{E_{m_{1}^{\prime}, m_{2}}\right\} \leq \varepsilon_{3}$ if $C_{1}^{\varepsilon}+C_{2}^{\varepsilon} \leq \Xi_{\min }^{\delta}\left(P_{X}\right)+\Xi_{\min }^{\delta}\left(P_{Y}\right)+\Xi_{\min }^{\delta}\left(P_{Z}\right)-$ $\Xi_{\max }^{\delta}\left(P_{X Y Z}\right)+\log \left(\varepsilon_{3}\right)$.

The above analysis shows that the average probability of error, averaged over all choices of codebooks in the random code construction achieves the error bound in 61. . Thus, there exists at least one code which achieves the desired error bound in 61 .

The asymptotic optimality of the above achievable rate pair for the case of memoryless multiple-access channel can be proved using ideas similar to that used in the proof of Lemma 1 and by invoking Definition 9 and Definition 10

\section{Conclusions And ACKNOWLEDGEMEnT}

In conclusion, we defined a novel one-shot typical set based on smooth entropies which helps us to get the one-shot rate region for distributed source coding and multiple access channel while leveraging the results from the asymptotic analysis. We parametrize this oneshot typical set using $\delta$. The choice of $\delta$, further depends upon the amount of error $(\varepsilon)$ that is allowed. In short, the decoding region is a function of $\delta$. Primarily, the motivations for the definition of this set is inspired by the definition of typical set for the asymptotic case and it turns out to be a serendipitous scenario where such a set has the desired properties asymptotically when large i.i.d. copies are available.

The authors wish to thank R. Renner for giving his helpful comments on the subject.

\section{REFERENCES}

[1] C. E. Shannon, "A mathematical theory of communication," Bell Sys. Tech. J., vol. 27, pp. 379-423 and 623-656, July and Oct. 1948.

[2] T. M. Cover and J. A. Thomas, Elements of Information Theory. Hoboken, NJ, USA: Wiley, 2nd ed., 2006.

[3] Y. Polyanksiy, H. V. Poor, and S. Vérdu, "Channel coding in the finite blocklength regime," IEEE Trans. Inf. Theory, vol. 56, pp. 2307-2359, May 2010.

[4] R. Renner and S. Wolf, "Smooth Rényi entropy and applications," in Proc. IEEE Int. Symp. Inf. Theory (ISIT), (Chicago, IL, USA), June 2004.

[5] R. Renner and S. Wolf, "Simple and tight bounds for information reconciliation and privacy amplification," in Advances in Cryptology-ASIACRYPT 2005, Lecture Notes in Computer Science, pp. 199-216, Springer-Verlag, 2005.

[6] C. Cachin and U. Maurer, "Smoothing probability distributions and smooth entropy," in Proc. IEEE Int. Symp. Inf. Theory (ISIT), (Ulm, Germany), June 1997.

[7] R. Renner, S. Wolf, and J. Wullschleger, "The single-serving channel capacity," in Proc. IEEE Int. Symp. Inf. Theory (ISIT), (Seattle, WA, USA), July 2006

[8] L. Wang, R. Colbeck, and R. Renner, "Simple channel coding bounds," in Proc. IEEE Int. Symp. Inf. Theory (ISIT), (Seoul, Korea), June 2009.

[9] R. Renner and R. König, "Universally composable privacy amplification against quantum adversaries," in Proc. Theory Crypt. Conf. (TCC), vol. 3378, pp. 407-425, Lecture Notes in Computer Science, Springer-Verlag, 2005.

[10] N. Datta and R. Renner, "Smooth Rényi entropies and the quantum information spectrum," IEEE Trans. Inf. Theory, vol. 55, pp. 28072815, 2009.

[11] R. König, R. Renner, and C. Schaffner, "The operational meaning of min- and max-entropy," IEEE Trans. Inf. Theory, vol. 55, pp. 4337-4347, Sept. 2009.

[12] F. Dupuis, P. Hayden, and K. Li, "A father protocol for quantum broadcast channels," IEEE Trans. Inf. Theory, vol. 56, pp. 29462956, June 2010.

[13] M. Berta, M. Christandl, and R. Renner, "The quantum reverse Shannon theorem based on one-shot information theory," Cоттип. Math. Phys., vol. 306, pp. 579-615, Sept. 2011.

[14] N. Datta and M.-H. Hsieh, "The apex of the family tree of protocols: optimal rates and resource inequalities," New J. Phys., vol. 13, p. 093042, Sept. 2011.

[15] J. M. Renes and R. Renner, "Noisy channel coding via privacy amplification and information reconciliation," IEEE Trans. Inf. Theory, vol. 57, pp. 7377-7385, Nov. 2011.

[16] B. Schoenmakers, J. Tjoelker, P. Tuyls, and E. Verbitskiy, "Smooth Rényi entropy of ergodic quantum information sources," in Proc. IEEE Int. Symp. Inf. Theory (ISIT), (Nice, France), June 2007.

[17] T. Holenstein and R. Renner, "On the randomness of independent experiments," IEEE Trans. Inf. Theory, vol. 57, pp. 1865-1871, April 2011.

[18] J. M. Renes and R. Renner, "One-shot classical data compression with quantum side information and the distillation of common randomness or secret keys." arXiv:1008:0452.

[19] V. Y. F. Tan and O. Kosut, "On the Dispersions of Three Network Information Theory Problems." arxiv:1201.3901, Jan. 2012.

[20] D. Slepian and J. Wolf, "Noiseless coding of correlated information sources," IEEE Trans. Inf. Theory, vol. 19, pp. 471-480, July 1973. 
[21] T. S. Han, Information-Spectrum Methods in Information Theory. Berlin, Germany: Springer-Verlag, 2003.

[22] A. Rényi, "On measures of entropy and information," in Proc. 4th Berkeley Symp. Math Stat. Prob., pp. 547-561, 1960.

[23] C. Bennett, G. Brassard, and U. M. Maurer, "Generalized privacy amplification," IEEE Trans. Inf. Theory, vol. 41, pp. 1915-1923, 1995.

[24] N. Sharma and N. A. Warsi, "One-shot Slepian-Wolf." arXiv:1112.1687, Jan. 2012. 